University of Vermont

UVM ScholarWorks

College of Arts and Sciences Faculty

Publications

College of Arts and Sciences

$9-1-2014$

\title{
Matrix models for quantifying competitive intransitivity from species abundance data
}

\author{
Werner Ulrich \\ Uniwersytet Mikołaja Kopernika w Toruniu \\ Santiago Soliveres \\ Universidad Rey Juan Carlos \\ Wojciech Kryszewski \\ Uniwersytet Mikołaja Kopernika w Toruniu \\ Fernando T. Maestre \\ Universidad Rey Juan Carlos \\ Nicholas J. Gotelli \\ University of Vermont
}

Follow this and additional works at: https://scholarworks.uvm.edu/casfac

Part of the Climate Commons

\section{Recommended Citation}

Ulrich W, Soliveres S, Kryszewski W, Maestre FT, Gotelli NJ. Matrix models for quantifying competitive intransitivity from species abundance data. Oikos. 2014 Sep;123(9):1057-70.

This Article is brought to you for free and open access by the College of Arts and Sciences at UVM ScholarWorks. It has been accepted for inclusion in College of Arts and Sciences Faculty Publications by an authorized administrator of UVM ScholarWorks. For more information, please contact scholarworks@uvm.edu. 
Oikos 123: 1057-1070, 2014

doi: 10.1111 /oik.01217

(C) 2014 The Authors. Oikos (C) 2014 Nordic Society Oikos

Subject Editor: Stefano Allesina. Accepted 4 February 2014

\title{
Matrix models for quantifying competitive intransitivity from species abundance data
}

\author{
Werner Ulrich, Santiago Soliveres, Wojciech Kryszewski, Fernando T. Maestre and \\ Nicholas J. Gotelli
}

W. Ulrich (ulrichw@umk.pl), Chair of Ecology and Biogeography, Nicolaus Copernicus Univ. in Toruń, Lwowska1, PL-87-100 Toruń, Poland. - S. Soliveres and F. T. Maestre, Área de Biodiversidad y Conservación, Depto de Biología y Geología, Escuela Superior de Ciencias Experimentales y Tecnología, Univ. Rey Juan Carlos, ES-28933 Móstoles, Spain. SS also at: Inst. of Plant Sciences, Univ. of Bern, Alterbengrain 21, CH-3013 Bern, Switzerland. - W. Kryszewski, Faculty of Mathematics and Informatics, Nicolaus Copernicus Univ. in Torun, Chopina 12/18, PL-87-100 Toruń, Poland. - N. J. Gotelli, Dept of Biology, Univ. of Vermont, Burlington, VT 05405, USA.

\begin{abstract}
In a network of competing species, a competitive intransitivity occurs when the ranking of competitive abilities does not follow a linear hierarchy $(\mathrm{A}>\mathrm{B}>\mathrm{C}$ but $\mathrm{C}>\mathrm{A})$. A variety of mathematical models suggests that intransitive networks can prevent or slow down competitive exclusion and maintain biodiversity by enhancing species coexistence. However, it has been difficult to assess empirically the relative importance of intransitive competition because a large number of pairwise species competition experiments are needed to construct a competition matrix that is used to parameterize existing models. Here we introduce a statistical framework for evaluating the contribution of intransitivity to community structure using species abundance matrices that are commonly generated from replicated sampling of species assemblages. We provide metrics and analytical methods for using abundance matrices to estimate species competition and patch transition matrices by using reverse-engineering and a colonization-competition model. These matrices provide complementary metrics to estimate the degree of intransitivity in the competition network of the sampled communities. Benchmark tests reveal that the proposed methods could successfully detect intransitive competition networks, even in the absence of direct measures of pairwise competitive strength. To illustrate the approach, we analyzed patterns of abundance and biomass of five species of necrophagous Diptera and eight species of their hymenopteran parasitoids that co-occur in beech forests in Germany. We found evidence for a strong competitive hierarchy within communities of flies and parasitoids. However, for parasitoids, there was a tendency towards increasing intransitivity in higher weight classes, which represented larger resource patches. These tests provide novel methods for empirically estimating the degree of intransitivity in competitive networks from observational datasets. They can be applied to experimental measures of pairwise species interactions, as well as to spatio-temporal samples of assemblages in homogenous environments or environmental gradients.
\end{abstract}

Species differ in their competitive ability, and these differences might translate to observed inequalities in species' relative abundances within multi-species assemblages (Meserve et al. 1996, Levine and Rees 2002). Ecologists have devoted much effort to inferring competitive processes from observed patterns of species abundances and morphology, and from changes in the spatio-temporal distribution of species (Gotelli and Graves 1996, Chesson 2000, Engel and Wetzin 2008). Such data are often summarized in a sample matrix A, in which rows represent species $i$, columns represent different sites $j$ (or multiple sampling times at a single site), and entries are the abundance or incidence of species $i$ at site (or time) $j$. Classic assembly rules models (Diamond 1975), derived from the principle of competitive exclusion (Gause 1934), predict that differences in competitive abilities should cause non-random patterns of species occurrences among sites and generate inequalities in species abundances within sites. Competitively inferior species are predicted to occur less frequently and at lower abundance, and an important and largely unresolved question is how such species can persist in a community over long time periods (Fox 2013).

Many theoretical models of competitive interactions assume that species can be ranked unequivocally $(\mathrm{A}>\mathrm{B}>\mathrm{C} \ldots>\mathrm{Z})$ according to their competitive strength or resource utilization efficiency (Tilman 1988). However, intransitive competitive networks (Gilpin 1975) can generate loops in the hierarchy of competitive strength (e.g. the rock-scissors-paper game, in which $\mathrm{A}>\mathrm{B}>\mathrm{C}>\mathrm{A}$ ). Theoretical and empirical studies have shown that competitive intransitivity can moderate the effects of competition, allowing weak competitors to coexist with strong ones (Huisman et al. 2001, Kerr et al. 2002, Laird and Schamp 2006, 2009, Reichenbach et al. 2007). The degree 
of intransitivity may change depending on environmental heterogeneity (Allesina and Levine 2011), successional stage (Worm and Karez 2002), or the presence of consumers (Paine 1984).

Despite the conceptual simplicity of intransitive competitive hierarchies, the empirical estimation of the strength of competition and of the frequency of competitive intransitivity in nature has proven difficult. Estimation is possible for small assemblages because researchers can perform separate competition experiments for every unique pair of $m$ species (Grace et al. 1993, Shipley 1993, Keddy and Shipley 1989). However, because there are $m(m-1) / 2$ such pairs, it quickly becomes impractical to test all species pairs for even a moderately-sized community. Perhaps as a consequence of this limitation, intransitivity as a driver of community structure (e.g. species diversity) has mainly been studied in a conceptual (Bowker et al. 2010, Bowker and Maestre 2012) and mathematical (May and Leonard 1975, Laird and Schamp 2006, 2009, Rojas-Echenique and Allesina 2011) framework, and existing models have rarely been applied to empirical data (but see Grace et al. 1993, Soliveres et al. 2011).

Theoretical work on the impact of intransitive competition hierarchies begins with the assumption that the structure and strength of the competitive networks are already known, but in real communities such competitive hierarchies are generally unknown (but see Miller and Werner 1987, Keddy and Shipley 1989 for examples). Instead, ecologists try to infer quantitative interactions from observed patterns of species incidences, abundances, or cooccurrences (Ovaskainen et al. 2010, Ulrich and Gotelli 2010). Such inferences are challenging, because replicated samples from real assemblages often exhibit multiple contrasting patterns of species co-occurrence that may be caused by multiple mechanisms, including biotic interactions such as competition and facilitation, and abiotic responses reflecting niche conservatism and habitat filtering (Gotelli and Ulrich 2012, Ulrich et al. 2012).

It is difficult, however, to infer the relative abundances of species in an assemblage at equilibrium from the entries in pairwise competition matrices, which are the ones used as the basis of most current modeling approaches (Engel and Wetzin 2008, but see Laird and Schamp 2006, 2008, 2009 for related approaches). Moreover, most current approaches assume competitive interactions to have deterministic dichotomous outcomes (win or lose). That is, for a set of $m$ species, competitive outcomes are coded in a binary $\mathrm{m} \times \mathrm{m}$ matrix. If the species in the row wins, the entry is coded as 1 , and if the species in the column wins, the entry is coded as 0 (Laird and Schamp 2006, Allesina and Levine 2011). This coding has proven especially useful to develop metrics of intransitivity such as the degree of nestedness (the progressive decrease of $1 s$ in the outcome matrix after ordering according to row and column totals; Laird and Schamp 2006) and to infer numerous consequences of changing the degree of intransitivity in ecological communities (Laird and Schamp 2006, Allesina and Levine 2011, Rojas-Echenique and Allesina 2011). However, deterministic competitive exclusion is uncommon even at equilibrium (Tilman 1994, Chesson 2000) and these binary pairwise competitive outputs are unlikely to represent competitive dynamics in natural communities. For these reasons, we prefer models of probabilistic competition outcomes, which may be more realistic, and allow for empirical tests with species abundance data.

In this paper, we propose metrics and statistical tests for evaluating the contribution of intransitivity and other patterns of competitive interaction to community structure in real communities. We develop a framework for the analysis of two types of data: competition matrices (hereafter $\mathbf{C}$ matrices) derived from the outcomes of pairwise experimental studies, and species abundance matrices (hereafter A matrices) derived from field samples that are replicated in time or space (Fig. 1). To translate empirical species abundance data into a competition matrix, we construct patch transition matrices (hereafter $\mathbf{P}$ matrices) and apply them in a simple Markov chain model.

The patch transition matrix describes the outcomes of species interactions at a small spatial scale in which a single individual occupies an entire patch. As in Horn's (1975) original exposition, the elements in this matrix reflect the outcomes of species interactions in each time step, which lead to either a species replacement in a patch, or continued occupancy by the original resident. Similar transition matrices are used in population genetics to predict equilibrium allele frequencies (Pritchard et al. 2000). In our analysis, the patch model provides a simple spatial representation

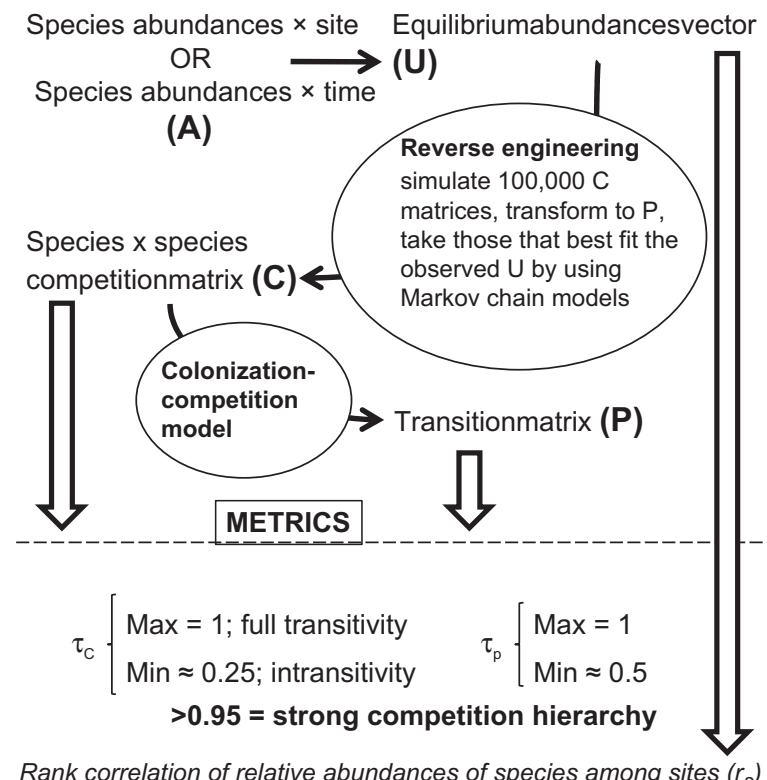

High values $=$ high dominance $=$ high transitivity

Figure 1. Flowchart illustrating the procedure followed to obtain the metrics of the degree of intransitivity from two different possible data sources (A [species abundance $\times$ sites or time] and $\mathrm{C}$ [pairwise competition] matrices). Three metrics are obtained from the measured/simulated matrices (A, C and P[patch transition]. The two main steps of our approach (reverse-engineering to estimate $\mathrm{C}$ from $\mathrm{A}$, and colonization-competition modeling to estimate $\mathrm{P}$ from $\mathrm{C}$ ) are shown within circles. The area below the dotted line details the three developed metrics to measure the degree of intransitivity within the observed community and how to interpret them. 
of interacting species that leads to changes in relative abundance. This spatial context is lacking in most theoretical models, which are based only on interaction coefficients.

In this way, we relate empirically-derived competition matrices $\mathbf{C}$ to an explicit colonization-interaction model to obtain patch transition matrices $\mathbf{P}$. Next, we use a 'reverse-engineering' approach to infer the structure of the competition $(\mathbf{C})$ and the transition $(\mathbf{P})$ matrices from an empirical (temporal or spatial) A matrix. There is no unique solution to this problem because a large number of different competition matrices $(\mathbf{C})$ can generate the same patch transition matrix $(\mathbf{P})$ that will reproduce the $\mathbf{A}$ matrix. However, by simulating a large set of stochastically created $\mathbf{C}$ matrices, the matrix that provides the best fit to an empirical A matrix can be analyzed with respect to its transitivity pattern. Finally, we develop and test different indices to quantify the degree of intransitivity from the underlying $\mathbf{C}$ and $\mathbf{P}$ matrices for different types of data. To illustrate our methods, we analyze empirical data matrices on the colonization of slug carrion by necrophagous flies and their parasitoids (Ulrich 1999).

\section{A matrix algebra approach to intransitivity}

Defining the pattern

Traditionally, researchers have organized experimentallyestimated measurements of pairwise competitive strengths among $m$ species as a square $m \times m$ matrix $\mathbf{C}$ in which an entry $c_{i j}$ is the probability that species $i$ replaces species $j$ in a competitive interaction. In such a matrix, the diagonal elements are set to 1.0, and therefore, such matrices cannot be used to directly predict species abundances from a Markov chain approach. However, the complementary matrix elements $c_{i j}$ and $c_{j i}$ always sum to unity because, for any pair of species, $c_{i j}=\left(1-c_{j i}\right)$. In models designed to test for the degree of intransitivity in a community (Laird and Schamp 2006), these $\mathbf{C}$ matrices are often transformed to dichotomous $0 / 1$ data (i.e. species $i$ completely replaces species $j$ or vice versa $\left[c_{i j}=1\right.$ or $\left.\left.c_{j i}=1\right]\right)$

The $\mathbf{C}$ matrix itself is not column stochastic (column sums do not sum to 1.0), so it cannot be used to estimate a vector of relative abundances in a Markov chain model without further information (for instance on resource use; Allesina and Levine 2011). To translate competitive strengths coded in the $\mathbf{C}$ matrix to species abundances, we need an additional patch matrix (P) that is column stochastic. The $\mathbf{P}$ matrix specifies the probability of transition from one species occupancy state to another, given the underlying competitive strengths in the $\mathbf{C}$ matrix.

\section{Transforming a competition matrix (C) to a transition matrix $(\boldsymbol{P})$}

A simple Markov chain model that predicts relative abundances is Horn's (1975) classic patch transition model. In this model, a $m \times m$ patch transition matrix $\mathbf{P}$ describes the probability $p_{i j}$ of a transition from a patch occupied by species $j$ to a patch occupied by species $i$ in a single time step. Here we adapt this model to predict relative abundances from competitive coefficients coded in C. Although the transitions in Horn's (1975) model are determined by the outcome of species interactions, their precise relationship to the elements of the $\mathbf{C}$ matrix is not clear. Horn's (1975) general model can also describe turnover in patch occupancy that is neutral or that reflects facilitation (McAuliffe 1988).

Assuming that the probabilistic outcome of species interactions is fully described by the entries of $\mathbf{C}$, we want to compute the patch transition matrix $\mathbf{P}$ in terms of the competition matrix $\mathbf{C}$. Here are the additional assumptions in our model (cf. Laird and Schamp 2008 for a similar model): 1) there are many homogeneous patches, each of which can be colonized and occupied by individuals of a set of $m$ species; 2) all species produce a large number of potential propagules, so there is a 'propagule rain', and colonization is never limited by dispersal limitation; 3) only a single species can occupy one patch at a time; 4) in a single time-step, a species occupying a patch either retains its occupancy or is replaced by a different species; 5) the set of patches is spatially unstructured.

A model satisfying the above assumptions might describe a species of plant or sessile invertebrate that competes with the mobile propagules of all potentially invading species. With this model, we can use simple probability rules to convert the entries in the $\mathbf{C}$ matrix into a $\mathbf{P}$ matrix. We denote the probabilities in the $\mathbf{P}$ matrix that species $i$ replaces species $j$ by $p_{i j}$. This definition makes the matrix $\mathbf{P}$ column stochastic, i.e. $\sum_{t=1}^{m} p_{i j}=1$.

For example, consider an assemblage of $m=$ three species $\{1,2,3\}$, with the $m \times m$ competition matrix $\mathbf{C}$. The off-diagonal entry $c_{12}$ in the $\mathbf{C}$ matrix specifies the probability that species 1 wins over species 2 . The probability $p_{11}$ that species 1 is not replaced by species 2 or 3 is given by

$p_{11}=p(1 \rightarrow 2) \wedge p(1 \rightarrow 3)=C_{12} C_{13}$

Diagonal entries for $p_{22}$ and $p_{33}$ are calculated the same way.

This result can be achieved differently: with probability $1 / 2$, species 1 meets species 2 , wins over it with probability $c_{12}$, then species 1 meets species 3 and with probability $c_{13}$ wins; or species 1 meets first species 3 with probability $1 / 2$ and wins over it with probability $c_{13}$ and then it meets species 2 winning over it with probability $\mathrm{c}_{12}$. Hence the total probability is

$p_{11}=\frac{1}{2} C_{12} C_{13}+\frac{1}{2} C_{13} C_{12}=C_{12} C_{13}$

The same logic can be applied to calculate the off-diagonal elements of the $\mathrm{P}$ matrix. Let us compute $\mathrm{p}_{12}$ for instance, i.e. the probability that species number 1 replaces the resident species 2 . With probability $1 / 2$ species 2 meets species 1 and loses with it with probability $\mathrm{c}_{12}$ (and, according to our assumption 4 , this ends the competition) or, with probability $1 / 2$ the resident species 2 meets species 3 , wins over it with probability $c_{23}$ (it cannot loose, since we compute the probability that species 2 is replaced by 1 ) and then meets 1 and loses it with probability $\mathrm{c}_{12}$. Hence

$p_{12}=\frac{1}{2} C_{12}+\frac{1}{2} C_{12} C_{23}$ 
In general the probability $p_{i j}$, with $1 \leq i \neq j \leq 3$, that species $j$ is replaced by the species $\mathrm{i}$ is calculated as

$p_{i j}=\frac{1}{2} C_{i j}+\frac{1}{2} C_{i j} C_{j k}$

and, for any $1 \leq i \leq 3$,

$p_{i i}=C_{i j} C_{i k}$

where $j, k \neq i$.

Generalizing to $\mathrm{m}>3$, consider $m=4$. For $1 \leq i \neq j \leq 4$ we have

$p_{i j}=\frac{1}{3} C_{i j}+\frac{1}{3}\left(\frac{1}{2} C_{i j} C_{j k}+\frac{1}{2} C_{i j} C_{j k} C_{j l}\right)+\frac{1}{3}\left(\frac{1}{2} C_{i j} C_{j l}+\frac{1}{2} C_{i j} C_{j l} C_{j k}\right)$

where $k \neq i, j, l$ and $l \neq i, j, k$, and for $1 \leq i \leq 4$

$p_{i i}=C_{i j} C_{i k} C_{i l}$

where $j, k, l \neq i$.

To generate the formula for $p_{i j}, i, j=1, \ldots, m$, for an arbitrary $m$, we need the following notation: given a set $\mathrm{A}=\left\{a_{1}, \ldots, a_{\mathrm{n}}\right\}$ of species with the corresponding competition matrix $\mathbf{C}$, let $\mathrm{P}(\mathrm{A})\left[a_{j} \rightarrow a_{i}\right]$ denote the probability that species $a_{\mathrm{j}}$ is replaced by $a_{i}, i, j=1, \ldots, n$.

Within this notation: for $1 \leq i \neq j \leq m$ and for $1 \leq i \leq m$

$$
p_{i i}=\prod_{j=1, j \neq i}^{m} C_{i j}+\frac{1}{m-1} \sum_{k=1, k \neq i}^{m} C_{i k} P(1, \ldots, k-1, k+1, \ldots, m)[i \rightarrow i]
$$

Equation 8 and 9 generate the required transition matrix $\mathbf{P}$ for an arbitrary number $m$ of species in terms of competitive strength matrices for sets consisting of $(m-1)$ species.

The fact that the transition probability for two species (Eq. 8) contains terms that include other species means that a fully transitive competitive strength matrix $\mathbf{C}$ is not necessarily transitive with respect to the transition matrix $\mathbf{P}$ (Fig. 2). A fully transitive $\mathbf{C}$ matrix translates into a transitive $\mathbf{P}$ matrix only if competitive strengths of the off-diagonal elements in $\mathbf{C}$ are either constant or increase in each row from left to right (Fig. 2, C2, C3). This feature is equivalent to a fully quantitatively nested pattern of competitive strength (cf. Staniczenko et al. 2013). If this ordering is broken, a transitive $\mathbf{C}$ matrix translates always into an intransitive $\mathbf{P}$ matrix (Fig. 2, C4). Thus, it is important to quantify intransitivity in both the $\mathbf{P}$ matrix and in the underlying $\mathbf{C}$ matrix. Importantly, full transitivity (when defined by transition probabilities) does not necessarily imply competitive exclusion. Only $\mathbf{C}$ matrices that translate into absorbing $\mathbf{P}$ matrices cause competitive exclusion (Fig. 2, C1).

We note that the dominant eigenvector of the simple Markov chain model predicts the relative abundances of all

$p_{i i}=P(1, \ldots, m)[j \rightarrow i]=\frac{1}{m-1} C_{i j}+\frac{1}{m-1} \sum_{k=1, k \neq i, j}^{m} C_{j k} P(1, \ldots, k-1, k+1, \ldots, m)[j \rightarrow i]$

Competitive strength matrix

Transition matrix

\begin{tabular}{|c|c|c|c|}
\hline (C) & A & B & C \\
\hline A & 1.00 & 1.00 & 1.00 \\
\hline B & 0.00 & 1.00 & 1.00 \\
\hline C & 0.00 & 0.00 & 1.00 \\
\hline
\end{tabular}

\begin{tabular}{|c|c|c|c|}
\hline (C) & A & B & C \\
\hline A & 1.00 & 0.80 & 0.80 \\
\hline B & 0.20 & 1.00 & 0.80 \\
\hline C & 0.20 & 0.20 & 1.00 \\
\hline
\end{tabular}

(P1) A $\quad$ B $\quad$ C

\begin{tabular}{|c|c|c|c|}
\hline A & 1.00 & 1.00 & 0.50 \\
\hline & 0.00 & 0.00 & 0.50 \\
\hline & 0.00 & 0.00 & 0.00 \\
\hline
\end{tabular}

Dominant

Eigenvector

(U1)

\begin{tabular}{|l|}
\hline 1.00 \\
\hline 0.00 \\
\hline 0.00 \\
\hline
\end{tabular}

(U2)

\begin{tabular}{|c|c|c|c|c|c|}
\hline & A & B & C & & (U2) \\
\hline$A$ & 0.64 & 0.72 & 0.48 & A & 0.63 \\
\hline & 0.18 & 0.16 & 0.48 & $\rightarrow \mathrm{B}$ & 0.22 \\
\hline & 0.18 & 0.12 & 0.04 & C & 0.15 \\
\hline
\end{tabular}

\begin{tabular}{|c|c|c|c|}
\hline (C) & A & B & C \\
\hline A & 1.00 & 0.60 & 0.80 \\
\hline$B$ & 0.40 & 1.00 & 0.60 \\
\hline C & 0.20 & 0.40 & 1.00 \\
\hline
\end{tabular}

(P3) A

(U3)

\begin{tabular}{|c|c|c|c|c|c|}
\hline 0.48 & 0.48 & 0.56 & & A & 0.50 \\
\hline 0.32 & 0.24 & 0.36 & & B & 0.32 \\
\hline 0.12 & 0.16 & 0.08 & & C & 0.18 \\
\hline
\end{tabular}

\begin{tabular}{|c|c|c|c|}
\hline (C) & A & B & C \\
\hline A & 1.00 & 0.80 & 0.30 \\
\hline B & 0.20 & 1.00 & 0.80 \\
\hline C & 0.70 & 0.20 & 1.00 \\
\hline
\end{tabular}

(P4) $A$

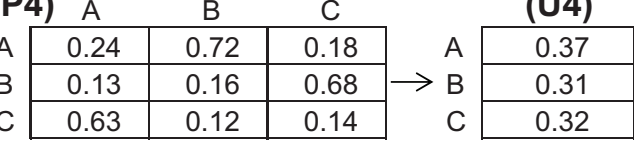

Figure 2. Four competitive strength matrices (C1 to $\mathbf{C 4}$ ), the corresponding column stochastic transition patch matrices $(\mathbf{P 1}$ to $\mathbf{P 4})$, and the respective dominant eigenvectors $\mathbf{U}$ that contain predicted equilibrium relative abundances generated from Eq. 8 and 9. Entries in the competitive strength matrices designate the probability that species A wins in pairwise competition against species B. Entries in the patch transition matrices (which are column stochastic) designate the probability that a patch occupied by species A is converted to a patch occupied by species B. Note that full competitive exclusion is only reached in case $\mathbf{C 1}$. 
species at equilibrium. This model implies that, whether or not the $\mathbf{P}$ matrix is dominated by transitive or intransitive chains, the more pairwise interactions in which a particular species wins, the greater its abundance at equilibrium (Allesina and Levine 2011). Therefore, a consistent rank order of species abundances among a set of sites that are environmentally similar implies a predominance of transitive species interactions. In contrast, an inconsistent ranking of species abundances among a set of similar sites would imply the existence of intransitive loops. Thus, the average degree of rank correlations of the relative abundances of across sites can be interpreted as a first metric of transitivity within the community (Fig. 1). A high evenness of species abundances in a single assemblage indicates some degree of intransitivity within a community.

\section{Estimating transitivity patterns with three data structures}

1) Temporal data

To estimate the degree of intransitivity in a given community, we need first to estimate the transition matrix $\mathbf{P}$ from an observed distribution of species abundances or occurrences. Depending on the data, there are three different scenarios. The first and most obvious approach relies on appropriate time series data. If $\mathbf{A}_{t}$ is the vector of relative abundances of $m$ species at generation $t$, then $\mathbf{P A}=\mathbf{A}_{t+1}$ if $\mathbf{P}$ is column stochastic. If data are available from at least $t+1$ time steps, the single abundance vectors of each generation can be embedded in two matrices $\mathbf{N}_{1, t}$, which runs from generation 1 to $t$, and $\mathbf{N}_{2, t+1}$, which runs from generation 2 to $t+1$. Combining these two matrices yields:

$\mathbf{P N}_{1, t}=\mathbf{N}_{2, t+1}$

and therefore

$\mathbf{P}=\mathbf{N}_{2, t+1} \mathbf{N}_{1, t}^{\mathrm{T}}\left(\mathbf{N}_{1, t} \mathbf{N}_{1, t}^{\mathrm{T}}\right)^{-1}$

where ${ }^{\mathrm{T}}$ denotes the transpose. This approach allows for the estimation of the $\mathbf{P}$ matrix from an $\mathbf{A}$ matrix of consecutive temporal censuses of an assemblage. Although a unique solution for $\mathbf{P}$ usually exists, in many cases the variability in species abundances not caused by competitive effects will return $\mathbf{P}$ matrices that are not column stochastic and that do not allow for an assessment of competitive interactions. Therefore we used a 'reverse-engineering' approach to find those $\mathbf{C}$ and $\mathbf{P}$ matrices that best mimicked observed abundances. For this task, we generated a large number $(\mathrm{n}=100000)$ of randomly assembled $\mathbf{C}$ matrices, in which each entry above the diagonal in the $\mathbf{C}$ matrix was chosen from a random uniform $(0,1)$ distribution. Using Eq. 8 and 9, we transformed the randomly assembled $\mathbf{C}$ matrices into $\mathbf{P}$ transition matrices to predict the $\mathbf{N}_{2, t+1}$ matrices from our Markov chain model. We used average rank order correlations between respective columns in the predicted and observed $\mathbf{N}_{2, t+1}$ matrices to assess goodness of fit, and selected those $\mathbf{P}$ and $\mathbf{C}$ matrices that generated the best fit to the observed vector of relative abundances $\left(\mathbf{A}_{t}\right)$. A worked example of this reversed-engineering approach is presented in the electronic supplement $\mathrm{C}$.
2) Spatial and environmental data

The second approach is based on spatial abundance data for $m$ species collected at $i=1$ to $n$ sites for which environmental variables are available. Assume a number of homogeneous patches. If observed species abundance distributions were determined only by competition, we could make a time-space substitution and interpret the vector $\mathbf{A}_{i}$ of the abundance distribution of $m$ species at site $i$ as representing the outcome of a single time step of a Markov chain triggered by the $m \times m$ matrix $\mathbf{P}$. At equilibrium, we can assume that the abundance distribution $\mathbf{A}_{i}$ at site $i$ (scaled to unity) approximates the right stable state vector (eigenvector) $\mathbf{U}(\mathbf{P U}=\boldsymbol{\Lambda} \mathbf{U})$, with $\boldsymbol{\Lambda}$ as the diagonal matrix of the largest eigenvalue $\lambda$. Thus it holds approximately

$\mathbf{P A}_{i} \approx \lambda \mathbf{A}_{i}$

We note that this eigenvector exists only if the matrix $\mathbf{P}$ defines an ergodic process, i.e. a process that converges to an equilibrium condition. But if $\mathbf{P}$ defines a periodic competitive hierarchy, no stable state vector exists, and species abundance distributions do not reach equilibrium. For instance, the intransitive competitive cycle $\mathrm{A}>\mathrm{B}>\mathrm{C}>\mathrm{A}$ is periodic. Although such a system does not reach stationary abundances, the distribution of abundances reaches an equilibrium. Using many single abundance vectors allows for the approximation of the generating probability matrix $\mathbf{P}$.

In the case of $n$ study sites, the single column vectors $\mathbf{A}_{i}$ form the $m \times n$ matrix $\mathbf{U}$ that contains the abundance distributions of the $m$ species among the $n$ sites. These can be viewed as individual approximations to the stable state vector $\mathbf{u}_{i}$. For large $n$ the average species abundance across the sites approximate the dominant eigenvector $\mathbf{U}$ (Allesina and Levine 2011) This is equivalent to

$\mathrm{PU} \approx \mathrm{U}$

Therefore, the problem of identifying the patch transition matrix $\mathbf{P}$ is reduced to the problem of solving $\mathbf{P}$ in Eq. 13. For this task, we decompose the variance in PU into a part explained by $\mathbf{U}$ and a part contained in an $m \times n$ matrix E to get the model

$\mathbf{P U}=\mathbf{U}+\mathbf{E}$

We next incorporate environmental data to estimate the matrix $\mathbf{E}$ from a multiple regression analysis. These data are contained in a $n \times b$ matrix $\mathbf{H}$, with $n$ sites (rows) and $h$ environmental variables (columns) measured at each site. The occurrence vector $\mathbf{B}_{i}$ of a species $i$ at the $n$ sites predicted by $\mathbf{H}$ is given by

$\mathbf{B}_{j}=\mathbf{H X}_{j}$

where $\mathbf{X}$ is the vector of regression parameters. Computed for all species, the regression model yields a predicted $n \times m$ matrix of species abundances that is identical to $\mathbf{B}^{\mathrm{T}}=\mathbf{H X}$, and that provides an estimate of that part of 
$\mathbf{U}+\mathbf{E}$ that is not explained by competition $\left[(\mathbf{H X})^{\mathrm{T}}=\mathbf{E}\right]$. This unexplained part can be plugged into Eq. 14 to yield

$\mathbf{P U}=\mathbf{U}+\mathbf{X}^{\mathrm{T}} \mathbf{H}^{\mathrm{T}}$

Therefore

$\mathbf{P}=\mathbf{I}+\mathbf{X}^{\mathrm{T}} \mathbf{H}^{\mathrm{T}} \mathbf{U}^{\mathrm{T}}\left(\mathbf{U} \mathbf{U}^{\mathrm{T}}\right)^{-1}$

with I being the $m \times m$ identity matrix. Equation 17 provides testable predictions about the competitive structure of the focal community. As with time series data, we use the 'reverse-engineering' approach and compare predicted and observed environmental terms $\mathbf{X}^{\mathrm{T}} \mathbf{H}^{\mathrm{T}}$ (Eq. 16) to find those $\mathbf{C}$ and $\mathbf{P}$ matrices that best mimic the observed abundance distributions. This method is only appropriate for relatively low levels of environmental variation. If the environmental variation is too great, the first assumption of the patch model is violated, and the calculation of the $\mathbf{C}$ matrices may not be valid.

\section{3) Spatial data}

Without additional environmental information for a precise definition of the minimum condition for E, Eq. 14 has no unequivocal non-trivial solution (Kryszewski pers. comm.). Therefore we use again the 'reversed-engineering' approach and assume that $\mathbf{P}$ predicts $\mathbf{U}$ best if the average rank correlations of all equivalent columns between predicted (PU) and observed (U) are above a predefined level $r_{\text {min }}$. Below, we use $r_{\min }=0.95$. We define this case as the minimum state of $\mathbf{E}=\mathbf{P U}-\mathbf{U}$. As before, we create a large number of randomly assembled $\mathbf{C}$ matrices, transform them into $\mathbf{P}$ matrices, and calculate $\mathbf{P U}$. The respective Spearman rank order correlations between equivalent matrix columns in the $\mathbf{P U}$ and $\mathbf{U}$ abundance matrices serve as a measure of goodness of fit. The best performing $\mathbf{C}$ and $\mathbf{P}$ matrices are then candidates for the competitive and transition matrices that generated the distribution of abundance in $\mathbf{U}$ (Supplementary material Appendix 1, C).

An important limitation of this method is that the final competition matrix does not necessarily coincide with a $\mathbf{P}$ matrix that produces the observed pattern of species abundances. If strong environmental heterogeneity and environmental filtering (Webb et al. 2002) influence species abundances, the matrix $\mathbf{E}$ will contain additional sources of variance. If environmental heterogeneity dominates the pattern of species abundances, $\mathbf{P}$ might underestimate the true strength of competition. Conversely, in cases where heterogeneity enhances disparities in abundance, $\mathbf{P}$ might overestimate the strength of competition. Both scenarios could bias the assessment of the importance of intransitivity. Thus a proper assessment of competitive hierarchies should be based on a homogeneous set of relatively similar study sites for which environmental variables do not contribute greatly to the variance contained in $\mathbf{E}$.

\section{Quantifying transitivity in C matrices}

Laird and Schamp (2006) proposed several useful metrics to quantify the degree of intransitivity in discrete $(0 / 1)$ C matrices. However, these indices do not work with $\mathbf{C}$ matrices that have probability entries $0<c_{i j}<1$. A simple measure of the degree of transitivity in probabilistic $\mathbf{C}$ matrices is the count $N$ of species pairs for which $c_{i j}<c_{j i}$ after the matrix has been sorted to maximize the number of matrix elements with $\mathrm{p}>0.5$ in the upper right triangle (Petraitis 1979). Although for each individual species pair $c_{i j}=\left(1-c_{j i}\right)$, the number of entries that end up above the diagonal after sorting should reflect the number of transitive chains in the matrix. The normalized version of this transitivity count $\left(\tau_{C}\right)$ is a quantitative measure of the degree of transitivity in $\mathbf{C}$ :

$$
\tau_{C}=\frac{2 N\left(c_{i j}<c_{j i}\right)}{m(m-1)}(i<j)
$$

\section{Quantifying transitivity in $\boldsymbol{P}$ matrices}

To quantify transitivity in the transition matrix $\mathbf{P}$, we propose the normalized count of the number of reversals in the decreasing order of probabilities for each column as an appropriate metric:

$\tau_{P}=1-\frac{2 N\left(p_{i j}<p_{k, j}\right)}{m(m-1)(m-2)}(i<k$ and $i, k \neq j)$

where $i$ and $j$ run from 1 to $m$ and $k$ from $i+1$ to $m$ ( $m$ is the total number of species in the studied community). Theoretically, this measure runs from 0.0 (completely intransitive) to 1.0 (fully transitive). However, because the entries in the $\mathbf{P}$ matrix are calculated from the probabilities in the $\mathbf{C}$ matrix, they are not independent of one another, and the matrix ordering of species according to the largest eigenvector imposes constraints on the lower boundary. In a simulation study of randomly filled $\mathbf{C}$ and $\mathbf{P}$ matrices, we found a rapid asymptotic convergence of the lower boundary towards $\tau_{P}=0.5$ with increasing species richness, with a minimum observed value $\tau_{C \text { min }}=0.25$ (cf. Petraitis 1979).

For both the $\mathbf{C}$ and the $\mathbf{P}$ matrices, another possible measure of intransitivity is $f_{\text {intr }}$, the fraction of species in the assemblage that participate in intransitive loops. However, this metric always tends to include a large number of species - even for moderately intransitive communities - and therefore cannot discriminate well among transitive and intransitive matrices. In nearly all test matrices for which $\tau_{P}$ and $\tau_{C}$ scores were less than 0.7 , all species took part in at least one intransitive relationship. We therefore used $f_{\text {intr }}$ as an auxiliary metric in cases of high, but imperfect, transitivity.

Finally we test for the use of the average Spearman rank order correlation $r_{S}$ between the abundance vectors $\mathbf{A}_{i}$. In this respect, Eq. 14 highlights an important property of the transition matrix P. If all abundance distributions $\mathbf{A}_{i}$ in the $n$ sites of $\mathbf{U}$ have the same ranking (all combinations of rank correlations among sites $=1.0$ ), the $\mathbf{P}$ matrix generates for all representations of $\mathbf{A}_{i}$ a constant abundance hierarchy. This occurs if the $\mathbf{C}$ matrix is highly (or even maximally) transitive. Conversely, the lower the average rank correlations within $\mathbf{U}$, the greater the degree of intransitivity in $\mathbf{P}$, and consequently also in $\mathbf{C}$. Therefore $r_{S}$ might also serve as an easily calculated metric of competitive transitivity (Fig. 1). 


\section{Methods}

\section{Benchmark testing of our approach}

For each of the three approaches described above, we first generated 200 competitive strength matrices $\mathbf{C}_{\text {test }}$ (with $m$ taken from a uniform random distribution: $5 \leq m \leq 50)$. Each matrix had a different predefined degree of intransitivity $\tau_{c}\left(0.7 \leq \tau_{c} \leq 1\right)$ implying that no $\left(\tau_{c}=1\right)$ or (nearly) all $\left(\tau_{c}<0.7\right)$ species were part of at least one intransitive loop. This specified degree of intransitivity serves as the true or known parameter, so the set of matrices can be used to test the accuracy of the procedures and metrics and their ability to correctly detect intransitivity.

For the time series approach, we generated $m<n \leq 50$ sequential abundance vectors by multiplying the species abundance distribution at time step $t$ with $\mathbf{P}_{\text {test }}$ to obtain the abundance distribution at time step $t+1$. The initial species abundance vector was drawn from an exponential random distribution. To introduce additional variability, all species abundances at each time step were multiplied by a uniform random number arbitrarily ranging between 0.8 and 1.2 . Thus, approximately $80 \%$ of the variance in a species relative abundance was determined by competitive interactions, and approximately $20 \%$ was random.

For the environmental and spatial approaches, we transformed these artificial $\mathbf{C}_{\text {test }}$ matrices into their respective $\mathbf{P}_{\text {test }}$ transition matrices (Eq. 8, 9), and calculated for each transition matrix the respective dominant eigenvector $\mathbf{V}_{\text {tess }}$. Non-ergodic $\mathrm{P}_{\text {test }}$ and the respective $\mathrm{C}_{\text {test }}$ matrices, which did not allow for the calculation of $\mathbf{V}_{\text {test }}$, were discarded. This eigenvector served as an estimate of the equilibrium distribution of the relative abundances of the species (Supplementary material Appendix 1, C). Proportional to this distribution, we randomly sampled individuals and assigned them to the elements of a matrix of $m$ species and $n$ sites ( $n$ again was sampled from a uniform random distribution: $5 \leq n \leq 50)$ until, at each site, all $m$ species were placed. This procedure yields for each site a normal approximation of the equilibrium abundance distribution and defines the required abundance matrix $\mathbf{U}_{\text {test }}$. For the environmental data approach, we also generated three environmental variables, each with values sampled from a random uniform $(0,1)$ distribution. Their sum served as an additional multiplicative source of variability for species abundances.

In the next step, we simulated for each $\mathbf{C}_{\text {test }}$ matrix $10 \times m \times n$, but not less than 100000 , random matrices with entries of the upper triangular matrix sampled from a uniform random $(0,1)$ distribution and translated each into a corresponding $\mathbf{P}$ matrix. To estimate the goodness of fit, we calculated for each of the random matrices the average rank order correlation between the columns of the $\mathbf{U}_{\text {test }}$ matrices $\left(\mathbf{N}_{2, \mathrm{~m}+1}\right.$ in the case of the time series approach) and the respective columns of the predicted $\left(\mathbf{U}_{\text {pred }}\right.$ and predicted $\mathbf{N}_{2, \mathrm{~m}+1}$ ) matrices (Supplementary material Appendix 1, C). We retained for each $\mathbf{C}_{\text {test }}$ matrix and its corresponding $\mathbf{P}_{\text {test }}$ matrix the 100 best-fitting matrices $\mathbf{C}_{\text {pred }}$ and $\mathbf{P}_{\text {pred. }}$. We used this set of best-fitting matrices to compare estimated and true patterns of intransitivity, and to identify the species with the strongest intransitivities in the $\mathbf{C}_{\text {test }}$ and $\mathbf{P}_{\text {test }}$ matrices. To infer how close the predicted matrices matched the respective simulated matrices, we directly compared the simulated and the (best-fitting) predicted $\mathbf{C}_{\text {test }}$ and $\mathbf{P}_{\text {test }}$ matrices using Mantel correlations applied to the respective Euclidean distance matrices. We also used these best-fitting matrices to compare the degrees of transitivity $\tau_{C}$ and $\tau_{P}$ that occurred in these matrices.

The calculation of the probabilities for all $\mathrm{p}_{i j}$ of the transition matrix $\mathbf{P}$ from the entries of the competition matrix $\mathbf{C}$ needs the evaluation of all combinations of $\mathrm{c}_{i k}$ $(k \neq i, j)$ according to the recursive Eq. 8 . This becomes computationally challenging at higher species richness. A good approximation of $\mathrm{p}_{i j}$ uses the fact that the calculation of each $\mathrm{p}_{i j}$ (Eq. 8) involves multiplications of all combinations of $\mathrm{c}_{i k}$ within each row $i$. Therefore, we might reduce Eq. 8 to a geometric series using the geometric average $\bar{x}$ of the respective $\mathrm{c}_{i k}$ values. This leads to

$p_{i j} \approx \frac{1}{m-1} C_{i j} \sum_{k-0}^{m-2} \bar{x}^{k}$

For our test matrices, the average relative error introduced by this approximation for $\mathrm{p}_{i j}$ was always less than $3 \%$ (not shown). Below we use this approximation to efficiently estimate $\mathbf{P}$ from $\mathbf{C}$.

All calculations were done with the FORTRAN software application 'Transitivity' (Ulrich 2013), which is available for free from the homepage of WU (<www.keib.umk.pl/ transitivity $/>$ ). The respective source code is contained in the Supplementary material Appendix 1, A.

\section{Case study}

To test our three methods, we used data from a controlled field experiment on the colonization of slug carrion Arion ater (Arionidae) by eight species of necrophagous Diptera and five species of oligophagous primary hymenopteran parasitoids of the Dipteran genus Megaselius (Phoridae) in a temperate German beech forest (Table 1; detailed description in Ulrich 1999). Carrion is permanently colonized by egg-laying females and thus resident and invading larvae strongly compete either for food or for phorid hosts. Such a system matches the assumptions of our patch model.

A total of 99 dead slugs were each assigned to one of nine weight classes $(2-3,3-4, \ldots, 9-10,12-19 \mathrm{~g}$ fresh weight) and exposed for 30 days in the field in polystyrol boxes, which allowed flies and parasitoids access, but excluded larger arthropod and vertebrate scavengers. In the same beech forest, the density of Hymenoptera parasitoids associated with the Diptera in this experiment was monitored for seven years (1981-1987; full description in Ulrich 2001). We used average densities (individuals $\times \mathrm{m}^{-2}$ ) for the first (spring) and the second (summer) generation of these parasitoids.

Additionally, we used data from a leaf-litter manipulation experiment conducted in 1986 to assess the influence of environmental variability on competitive strength (full description in Ulrich 2001). In this experiment, leaf litter in two experimental plots was either totally or partially removed and on two other plots was supplemented two- or fivefold (Ulrich 2001). Collectively, these data provide all the information necessary to calculate the three proposed 
Table 1. Numbers of correctly identified test matrices of a predefined degree of transitivity $\left(\tau_{p}\right.$ and $\left.\tau_{C}\right)$ in benchmark tests based on spatial, time series, and environmental data. $\mathrm{N}$ denotes the total number of simulated matrices in each category (from a total of 200 test matrices), and 'identified' gives the number of tests for which the upper $95 \%$ confidence limit of the 100 best-performing reverse-engineered $\mathbf{P}$ or $\mathbf{C}$ matrices included the value 1.0 (predefined $\tau_{C}, \tau_{P}=1$ ) or excluded the value 1.0 (predefined $\tau_{C}, \tau_{P}<1$ ).

\begin{tabular}{|c|c|c|c|c|c|c|c|c|c|}
\hline \multirow[b]{2}{*}{ Degree of transitivity } & \multicolumn{3}{|c|}{ Time series } & \multicolumn{3}{|c|}{ Environmental data } & \multicolumn{3}{|c|}{ Spatial data } \\
\hline & $\mathrm{N}$ & Identified & $\%$ identified & $\mathrm{N}$ & Identified & $\%$ identified & $\mathrm{N}$ & Identified & $\%$ identified \\
\hline \multicolumn{10}{|l|}{$\mathbf{P}$ matrices } \\
\hline$\tau_{P}=1$ & 20 & 9 & 45 & 25 & 9 & 36 & 30 & 18 & 60 \\
\hline $0.95 \leq \tau_{P}<1$ & 27 & 20 & 74 & 33 & 31 & 94 & 43 & 40 & 93 \\
\hline$\tau_{P}<0.95$ & 153 & 144 & 94 & 142 & 142 & 100 & 127 & 127 & 100 \\
\hline \multicolumn{10}{|l|}{ C matrices } \\
\hline$\tau_{C}=1$ & 40 & 20 & 50 & 33 & 20 & 61 & 35 & 17 & 49 \\
\hline $0.95 \leq \tau_{C}<1$ & 18 & 13 & 72 & 20 & 18 & 90 & 16 & 13 & 81 \\
\hline$\tau_{C}<0.95$ & 142 & 114 & 80 & 148 & 130 & 88 & 149 & 131 & 88 \\
\hline
\end{tabular}

methods for the same set of species within a single habitat. To estimate transitivity, we used the summed biomasses of each of the Diptera species, parasitism rates for the parasitoids in the slug experiment, and the abundances for the parasitoids in the time series data set. The complete raw data are provided in the Supplementary material Appendix 1, B.

To assess the degree of community-wide negative species associations, we used the incidence based C-score (Stone and Roberts 1990) and its abundance-based equivalent, $C A$ (Ulrich and Gotelli 2010). Both metrics need to be compared with a null model that provides an appropriate random expectation (Gotelli and Ulrich 2012, Ulrich and Gotelli 2013). For the Diptera and hymenopteran parasitoids, the individual slugs represent sites, which differed in size and desiccation rate. The different Diptera and Hymenoptera species also differed in their local abundances and incidences, which probably reflects differences in colonization potential.

To account for these typical sources of heterogeneity among sites and species, we used the fixed abundances null model IT of Ulrich and Gotelli (2010), which was developed for the randomization of abundance matrices. The IT algorithm assigns individuals randomly to matrix cells with probabilities proportional to observed row and column abundance totals until, for each row and column, total abundances are reached. Null expectations and standard deviations of the IT null distributions were in all cases based on 200 randomizations for each matrix. All cooccurrence analyses were conducted with the FORTRAN software application 'Turnover' (Ulrich 2011, Ulrich and Gotelli 2013). Because null distributions appeared to be approximately normal, we used the standardized effect sizes (SES, calculated from the observed score $x$ and the mean $\mu$ and standard deviation $\sigma$ of the null distribution as: $S E S=(x-\mu) / \sigma)$ to infer statistical significance. For a twotailed 95\% confidence interval, 'random' SES scores should range between \pm 1.96 .

\section{Results}

\section{Benchmark testing}

We found highly significant correlations between simulated and predicted degrees of transitivity, regardless of the approach used to recover competitive interactions from abundance data (Fig. 3). Our 'reverse-engineering' algorithm performed best for $\mathbf{P}$ matrices in combination with the spatial and environmental data (Fig. 3). In these analyses, the regression of estimated versus true transitivity explained $94 \%$ of the variance found in the data. Our methods were less successful at estimating pairwise competitive strength, and the respective regressions explained only between 51\% (abundance data, Fig. 3F) and 53\% (time series data, Fig. 3B) of the variance.

Despite variability in the prediction of the precise degree of transitivity, all three approaches were able to identify at least moderate degrees of intransitivity in test matrices (Table 1). For $\mathbf{P}$ matrices, each of our three approaches correctly recovered more than 94\% (time series approach) of the moderately to highly intransitive test matrices, with $\tau_{p}<0.95$ (Table 1 ). For $\mathbf{C}$ matrices, at least $80 \%$ (times series) of them were correctly identified. Of the weakly intransitive matrices $\left(0.95<\tau_{p}<1.0\right)$ between 74\% (time series) and 94\% (environmental data) were identified as being intransitive by the $\mathbf{P}$ matrices, and between 72\% (time series) and 90\% (environmental data) of them were identified as being intransitive by the C matrices.

These methods were less successful in identifying perfectly transitive matrices (Table 1). For $\mathbf{P}$ matrices, between 36\% (environmental data) and 60\% (spatial data) of the upper $95 \%$ confidence limits of the $\tau_{p}$ distributions of the 100 best-performing matrices included the value of 1.0 (full transitivity). For $\mathbf{C}$ matrices, between 49\% (spatial data) and $61 \%$ (environmental data) were correctly identified as transitive. In all of the fully transitive test matrices, the predicted transitivity scores of the best-performing engineered $\mathbf{P}$ and $\mathbf{C}$ matrices was $>0.95$ (not shown). Therefore, values of $\tau_{P}$ or $\tau_{C}>0.95$ might serve as a strong indicator of full transitivity.

A direct comparison of the simulated and predicted entries of the $\mathbf{P}$ and $\mathbf{C}$ matrices (Fig. 3G-H) revealed in all cases a high correlation, which increased with the degree of transitivity. The correlation coefficient (Mantel test) between the simulated and the best-fitting matrices was extremely high $\left(\mathrm{r}_{\text {interaction }}>0.95\right)$ in $88 \%$ of the fully transitive interaction matrices, indicating a high match between the simulated data and the matrices predicted with our approach (Fig. 3G). 

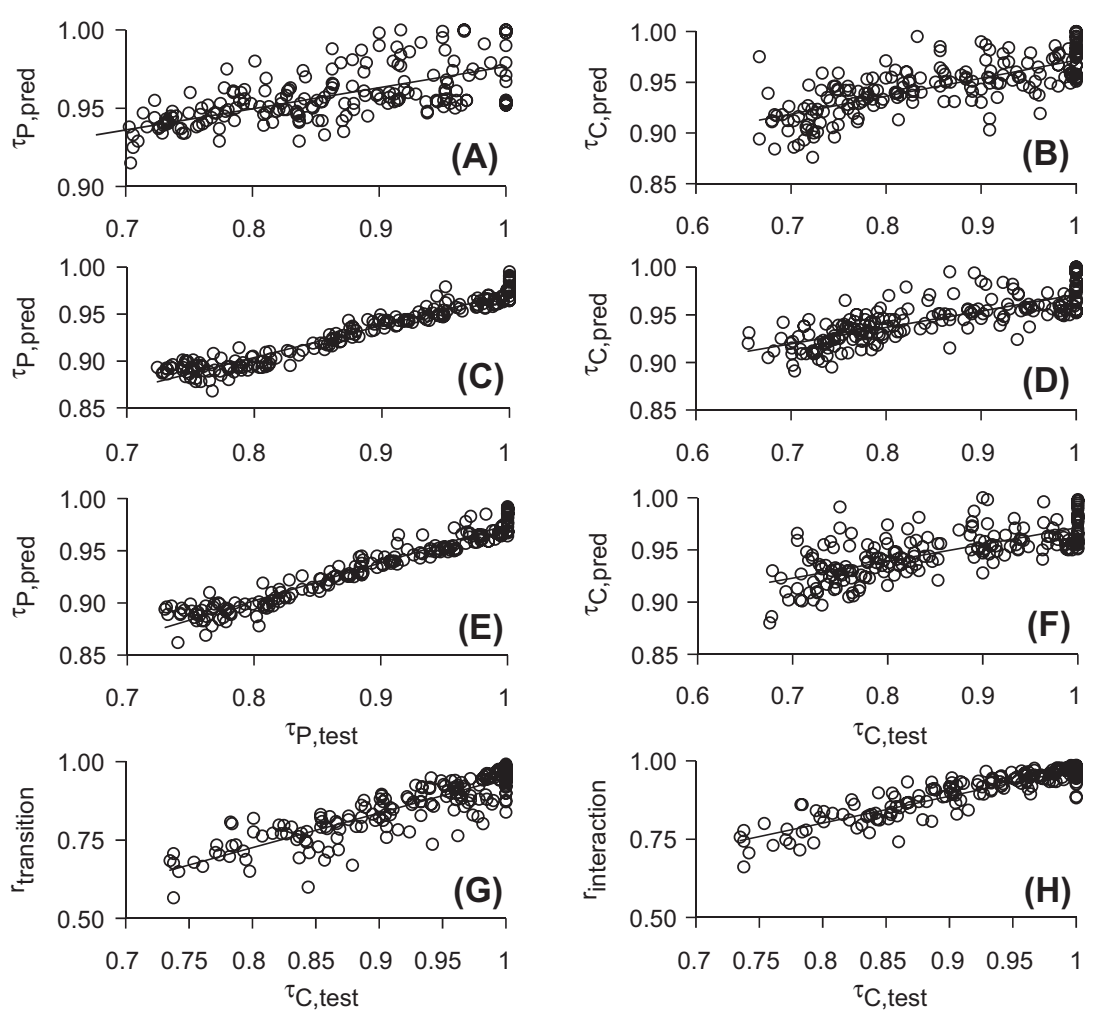

Figure 3. Simulated $\left(\tau_{P \text { test }}, \tau_{C \text {,test }}\right)$ and predicted $\left(\tau_{p_{\text {pred }}}, \tau_{C \text { pred }}\right)$ degrees of transitivity of competitive strength $\mathbf{C}$ and transition $\mathbf{P}$ matrices for the time series method (A, B), the environmental correlation method (C, D), and the spatial method (E, F). G, H: Mantel test correlation coefficients (Euclidean distances) of simulated and predicted interaction $\left(\mathrm{r}_{\text {interaction }}\right)$ and transition $\left(\mathrm{r}_{\text {transition }}\right)$ matrices in dependence on $\tau_{C \text {,test }}$. Each point represents the scores from a different simulated matrix $\left(n=200\right.$ matrices). Regression lines: A: $r^{2}=0.44 ; B: r^{2}=0.53$; C: $r^{2}=0.94 ; D: r^{2}=0.60 ; E: r^{2}=0.94 ; F: r^{2}=0.51 ; G: r^{2}=0.78 ; H: r^{2}=0.84(p<0.01$ in all cases).

\section{Case study}

The eight dipteran and five hymenopteran species differed markedly in abundance, biomass, and parasitism rates (Table 2). Abundances of Diptera and Hymenoptera ranged between 0.2 and 52 individuals per slug, which corresponds to 19 and 5186 individuals of the least and most abundant species, respectively. Irrespective of carrion weight class, species incidences and abundances of both taxa tended to be significantly segregated (Fig. 4), suggesting a predominance of negative species interactions. The degree of segregation increased with slug weight class (Fig. 4).

Table 2. Average densities ( $D$, individuals per slug), dry biomasses (B, mg dry weight per slug), and parasitism rates $p$ of eight necrophagous Diptera and five primary parasitoids of Megaselia ruficornis and M. pulicaria. Errors denote plus or minus one standard deviation. Average rank and range of ranks give the mean and the range of species ranks in the competitive hierarchies of the ten carrion weight classes.

\begin{tabular}{lcccc}
\hline Species & $\mathrm{D}$ & $\mathrm{B}$ & Average rank & Range of ranks \\
\hline Diptera & & & 2.2 & $1-6$ \\
Conicera schnittmani & $51.9 \pm 80.4$ & $0.030 \pm 0.051$ & 4.6 & $2-8$ \\
Fannia immuntica & $1.0 \pm 2.8$ & $0.004 \pm 0.012$ & 3.8 & $2-8$ \\
Gymnophora arcuata & $0.6 \pm 1.8$ & $0.001 \pm 0.004$ & 4.7 & $1-8$ \\
Limosina sp. & $23.2 \pm 34.1$ & $0.014 \pm 0.020$ & 5.3 & $2-8$ \\
Megaselia pulicaria & $6.7 \pm 13.9$ & $0.007 \pm 0.015$ & 5.0 & $1-8$ \\
Megaselia ruficornis & $8.2 \pm 9.3$ & $0.009 \pm 0.010$ & 5.8 & $4-8$ \\
Panorpa sp. & $0.4 \pm 0.9$ & $0.008 \pm 0.021$ & 4.6 & $2-8$ \\
Psychoda sp. & $3.3 \pm 7.3$ & $0.003 \pm 0.007$ & Average rank & Range of ranks \\
\hline Species & $\mathrm{D}$ & $\mathrm{P}$ & 1.6 & $1-2$ \\
\hline Hymenoptera & & & 2.0 & $1-3$ \\
Aspilota A & $3.4 \pm 5.8$ & $0.22 \pm 0.30$ & 4.0 & $3-5$ \\
Aspilota B & $3.4 \pm 8.1$ & $0.18 \pm 0.28$ & 4.9 & $4-5$ \\
Aspilota C & $0.5 \pm 1.4$ & $0.04 \pm 0.16$ & 2.5 & $1-4$ \\
Aspilota D & $0.2 \pm 1.1$ & $0.01 \pm 0.07$ & & \\
Orthostigma sp. & $2.7 \pm 4.4$ & $0.17 \pm 0.28$ & & \\
\hline
\end{tabular}



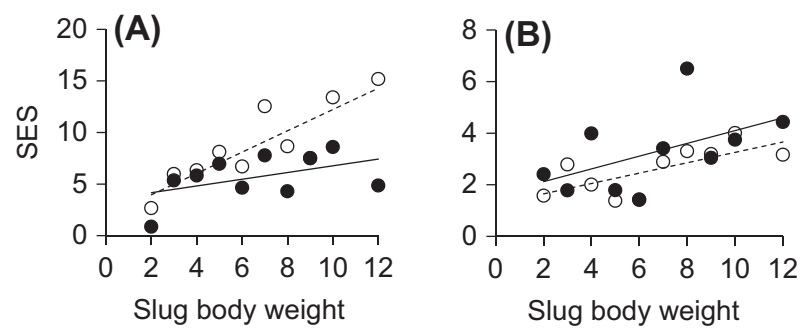

Figure 4. The degree of species segregation of necrophagous Diptera (A) and their parasitic Hymenoptera (B) among ten slug weight classes as quantified by the presence-absence based $C$-score (full circles and regression line; $A: r=0.47, p=0.17$; $\mathrm{B}: \mathrm{r}=0.52, \mathrm{p}<0.13)$ and the abundance based $C A$ score (open circles and broken regression line; A: $r=0.86, p<0.01$; $\mathrm{B}: \mathrm{r}=0.70, \mathrm{p}<0.02)$ increased with slug body weight.

The average predicted degree of transitivity of the Diptera community was $\tau_{p}=0.99$ and $\tau_{C}=0.99$, respectively (Table 2). The level of transitivity did not change with carrion weight class $(r=0.13, \mathrm{p}>0.1)$ or with the degree of negative species association $(\mathrm{r}=-0.12, \mathrm{p}>0.1$, Table 2$)$. Five of the predicted $\tau_{P}$ scores and all predicted $\tau_{C}$ scores were not significantly different from 1.0 , a score which indicates full transitivity (Table 3). Thus competitive hierarchies within the fly communities appeared to be fully, or at least nearly fully, transitive. In accordance with our probabilistic interpretation of pairwise species interactions, the degree of transitivity differed between the pairwise

Table 3. $\tau_{p}$ and $\tau_{C}$ (given as the average transitivity metrics based on the 100 best fit transition and competition matrices according to Eq. 18 and 19) for species $\times$ slug carrion matrices of 10 carrion weight classes (in g). $P_{p}(1.0)$ and $P_{C}(1.0)$ give the probabilities that the distribution of $\tau_{p}$ and $\tau_{C}$ based on the 100 best-fitting $\mathbf{P}$ an C matrices include the fully transitive pattern of $\tau_{p}=1.0$ and $\tau_{C} \tau 1.0$.

\begin{tabular}{ccccc}
\hline Slug weight class & $\tau_{P}$ & $\mathrm{P}_{\mathrm{p}}(1.0)$ & $\tau_{C}$ & $\mathrm{P}_{\mathrm{C}}(1.0)$ \\
\hline Diptera & & & & \\
2 & 0.998 & $>0.5$ & 0.999 & $>0.5$ \\
3 & 0.968 & 0.01 & 0.980 & $>0.5$ \\
4 & 0.946 & 0.01 & 0.934 & 0.08 \\
5 & 0.985 & 0.03 & 0.989 & $>0.5$ \\
6 & 0.988 & 0.08 & 0.993 & $>0.5$ \\
7 & 0.987 & 0.08 & 0.989 & $>0.5$ \\
8 & 0.990 & 0.14 & 0.989 & $>0.5$ \\
9 & 0.975 & 0.01 & 0.975 & 0.47 \\
10 & 0.998 & $>0.5$ & 0.999 & $>0.5$ \\
12 & 0.972 & 0.01 & 0.978 & $>0.5$ \\
All & 0.985 & 0.08 & 0.988 & $>0.5$ \\
Hymenoptera & & & & \\
2 & 0.991 & $>0.5$ & 0.900 & 0.01 \\
3 & 0.995 & $>0.5$ & 0.897 & 0.01 \\
4 & 0.972 & 0.32 & 0.993 & $>0.5$ \\
5 & 0.987 & $>0.5$ & 0.899 & 0.01 \\
6 & 0.999 & $>0.5$ & 1.000 & $>0.5$ \\
7 & 1.000 & $>0.5$ & 0.900 & 0.01 \\
8 & 0.939 & 0.02 & 0.904 & 0.05 \\
9 & 0.944 & 0.13 & 0.891 & 0.01 \\
10 & 0.952 & 0.22 & 0.970 & $>0.5$ \\
12 & 0.880 & 0.01 & 0.951 & $>0.5$ \\
All & 0.944 & 0.140 & 0.968 & $>0.5$ \\
\hline
\end{tabular}

competitive strength $(\mathbf{C})$ and the transition $(\mathbf{P})$ matrices, with the latter showing a stronger pattern of intransitivity (Table 3).

For Hymenoptera, there was a trend towards increasing intransitivity $\left(\tau_{p}\right)$ at higher slug weight $(\mathrm{r}=-0.82, \mathrm{p}<0.01$, Table 2). The confidence limits of $\tau_{p}$ of the 8 and $12 \mathrm{~g}$ carrion weight classes did not encompass 1.0 (Table 3). $\tau_{p}$ was also negatively correlated with the degree of species segregation $(\mathrm{r}=-0.78, \mathrm{p}<0.01$, Table 3$)$. This trend was not obvious for $\tau_{C}$ (Table 3 ).

Detailed comparisons of the competitive hierarchies of flies and parasitoid wasps (Table 2), revealed a reordering of species competitive strength between the different carrion weight classes. The average coefficient of correlation between all 45 combinations of predicted species rank orders of competitive strength was $r=0.11$ for Diptera and $r=0.76$ for Hymenoptera. Predicted average rank order of competitive strength of both taxa was negatively correlated with the respective average species abundance (Fig. 5), although this trend was not significant at $p<0.05$ for the Diptera. In accordance with the observed reordering of competitive strength across slug weigh classes, we found for both taxa the degree of transitivity within each slug weight class to be uncorrelated with the average Spearman rank order correlation between the respective biomass, parasitism rate, and abundance ranks (Fig. 6).

Both time series (spring and autumn generations) and the data from the leaf manipulation experiment pointed to fully transitive competitive relationships among the five parasitoid species (Table 4). Again, we observed differences in the competitive hierarchy between generations and between the time series and the leaf litter experiment.

\section{Discussion}

A central question in community ecology is how diversity is maintained among a set of competing species. Together with niche differentiation and neutral theory (Tilman

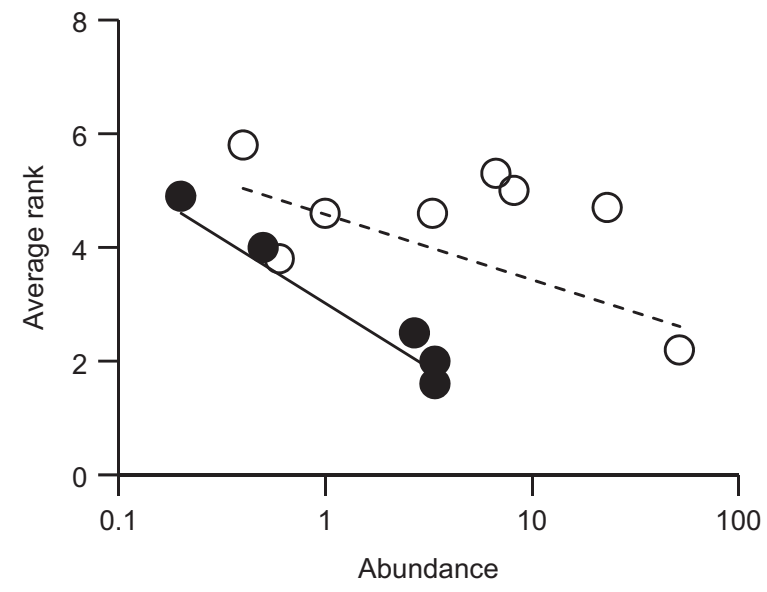

Figure 5. The average rank in the competitive hierarchies among necrophagous Diptera (open circles) and their parasitic Hymenoptera (full circles) decreases with average abundance among the slug carrions. Diptera: $\mathrm{r}=-0.76, \mathrm{p}=0.03$; Hymenoptera: $\mathrm{r}=-0.98$, $\mathrm{p}<0.01$. 

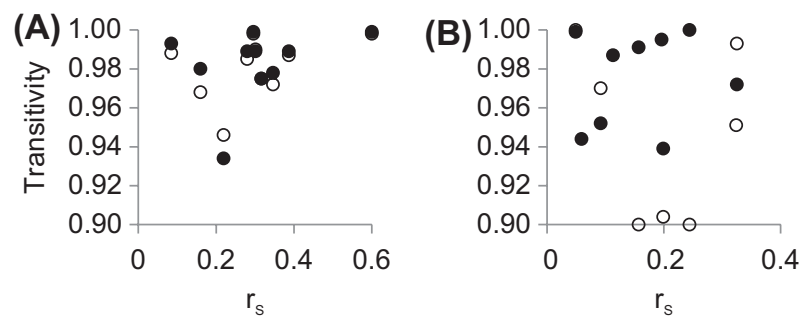

Figure 6. The degree of transitivity (full circles $\tau_{C}$ and open circles $\left.\tau_{p}\right)$ within each of the ten slug weight classes did neither for necrophagous Diptera (A) nor for their parasitic Hymenoptera (B) significantly $(\mathrm{p}<0.1)$ depend on the average Spearman rank order correlations among Diptera species body weights (A) and Hymenoptera parasitism rates (B). Using abundances instead of body weights and parasitism rates did not qualitatively change this result (not shown).

1994, Chesson 2000, Hubbell 2001), empirical (Bowker et al. 2010), mathematical (Laird and Schamp 2006), and theoretical (Paine 1984) research has pointed to intransitivity in competition networks (Gilpin 1975) as a key mechanism for the maintenance of diversity in natural communities (but see Shipley 1993). However, assessing the relative importance of intransitive competition networks in the field has been very difficult because it requires a large number of pairwise competition experiments linked to observed abundances of the interacting species, something extremely rare to find (Silvertown and Dale 1991, Engel and Weltzin 2008, Allesina and Levine 2011). This is likely the reason why empirical research focused on this topic is very scarce, despite the fact that it was introduced almost 40 years ago by Gilpin (1975).

The approach introduced here overcomes this problem by estimating competition hierarchies - and their associated degree of intransitivity - among the interacting species from their observed abundances in the field, rather than from direct measurements of interaction coefficients from pairwise competition experiments. We developed three methods for reconstructing pairwise competitive strength matrices $\mathbf{C}$ using intermediate patch transition matrices $\mathbf{P}$. Because $\mathbf{C}$ matrices code all pairwise interactions between the species involved in a competitive network, they are preferable from a theoretical point of view. However, there are so many entries in a typical $\mathbf{C}$ matrix that it may be difficult to estimate them from pairwise competition experiments. $\mathbf{P}$ matrices can be more easily estimated from repeated samples of an assemblage of potential competitors,

Table 4. Time series and environmental data measuring the degree of transitivity in the hymenopteran parasitoids of Megaselia flies. $\tau_{p}$ and $\tau_{C}$ are the average transitivity metrics based on the 100 best-fitting transition and competition matrices (Eq. 11 and 12). $P_{P}(1.0)$ and $P_{C}(1.0)$ give the probabilities that the distribution of $\tau_{P}$ and $\tau_{C}$ based on the 100 best fitting $\mathbf{P}$ an $\mathbf{C}$ matrices includes the fully transitive pattern of $\tau_{P}=1.0$ and $\tau_{C}=1.0$.

\begin{tabular}{lcccc}
\hline Variable & $\tau_{P}$ & $\mathrm{P}_{\mathrm{P}}(1.0)$ & $\tau_{C}$ & $\mathrm{P}_{\mathrm{C}}(1.0)$ \\
\hline First generation & 0.963 & 0.01 & 1.000 & 0.50 \\
Second generation & 0.998 & 0.50 & 1.000 & 0.50 \\
Both generations & 1.000 & 0.50 & 1.000 & 0.50 \\
Environment & 1.000 & 0.50 & 1.000 & 0.50 \\
\hline
\end{tabular}

such as successional series. However, if conditions change through time (as in classic succession models; Connell and Slatyer 1977), the $\mathbf{P}$ matrix entries will be affected by both species interactions and abiotic conditions in each time step (Zaplata et al. 2013).

It is possible to construct other more complicated patch transition models, such as cellular automata models (Baltzer et al. 1998), but these models would require even more assumptions. Horn's (1975) patch transition model is the simplest way to convert the effects of pairwise species interactions into changes in relative abundance. One important caveat for this patch model is that it assumes the outcomes of species interactions are density independent. This assumption is not unreasonable for many sessile invertebrates and plants that produce large quantities of mobile larvae or seeds and act effectively as a 'propagule rain'. This colonization scenario underlies classic models of island biogeography and metapopulation dynamics (Gotelli 2008).

As revealed by our benchmark testing, the methods introduced here successfully identify candidate competition matrices that predict abundance distributions that are very similar to the observed ones. Our approach recovers competitive hierarchies (Fig. 3), and intransitive test matrices always had predicted $\tau_{P}$ and $\tau_{C}$ values $<0.95$. Thus, we propose this 0.95 value as a rule of thumb to separate communities with a strong transitive hierarchy in their competitive networks from those showing some degree of intransitivity (Fig. 1). Environmental heterogeneity can override these patterns (Fig. 3), but a pattern of consistent species rank abundances among sites is always a strong indicator of a high degree of competitive transitivity. However, the converse is not true. If species ranks vary widely among sites, it could indicate either the presence of intransitive networks and/or environmental heterogeneity among sites.

Our approach characterizes transitive hierarchies only in terms of simple pairwise species interactions. However, complex multi-species interactions and indirect effects may alter the outcome of pairwise interactions by at least two different mechanisms: 1 ) even in a completely transitive pairwise network $(A>B, A>C, B>C)$, species $A$ might enhance the performance of species $C$ by suppressing species $\mathrm{B}$, and thus generate a competitive loop (indirect positive interactions; Levine 1999). These indirect positive interactions could also likely reduce competitive exclusion of the weakest species, and thus affect community-wide competitive networks. Complex outcomes are possible if subsets of species larger than just a species pair interact, and if, in pairwise contests, species A may outcompete species B and species $\mathrm{C}$, but species $\mathrm{B}$ and species $\mathrm{C}$ might jointly outcompete A (i.e. additive competitive effects, Chesson 2000). The effects of such additive competition on competition networks are likely to mimic those of indirect facilitative interactions.

Our approach is a first step towards disentangling the possible interactions in multispecies competitive situations by complementary using both $\mathbf{C}$ and $\mathbf{P}$ matrices, while providing testable hypotheses for models of bivariate competitive interactions (the pairwise outcomes in the simulated $\mathbf{C}$ matrices). We must note, however, that species interactions, even in small communities, are by far too complex to be 
described using single metrics. Our approach does not aim to precisely describe all pairwise competitive relationships. Rather, it indicates whether a focal community is predominately transitive or intransitive, and points to possible candidate species responsible for intransitivity loops. These predicted interactions might be verified in subsequent controlled experiments.

A common but unrealistic assumption of many intransitivity analyses conducted to date is that only negative, competitive interactions are important (Laird and Schamp 2006, Allesina and Levine 2011). However, positive (or facilitative) interactions are ubiquitous, not only among plants (Callaway 2007), but also among many other taxa (Kawai and Tokeshi 2007, Fugère et al. 2012). Facilitative interactions can increase the degree of intransitivity in a given community in two ways: 1) by increasing the number of species that can colonize a given site (Lortie et al. 2004), as this increases the chance of finding an intransitive network (Laird and Schamp 2008), and 2) by increasing the heterogeneity in the spatial distribution of resources, which can permit the coexistence of weak competitors (Allesina and Levine 2011). Although we can incorporate these positive interactions with our method by applying it to contrasting microsites (e.g. nurse plant vs open areas; Soliveres et al. 2011), future studies should explicitly include positive interactions directly into pairwise competitive strength $(\mathbf{C})$ or transition probability $(\mathbf{P})$ matrices.

\section{Case study}

Our case study revealed differences in the structure of competitive hierarchies of communities of necrophagous flies and their hymenopterous parasitoids. For the Diptera, the most probable pattern was complete transitivity. The hymenopteran parasitoids were characterized by transitive hierarchies at lower carrion weight classes, and a tendency towards intransitive loops at higher weight classes (Table 2). We note, however, that we do not have independent pair-wise control experiments to verify this prediction.

Contrary to our expectation, we did not find a strong relationship between the degree of transitivity and the stability of hymenopteran and dipteran abundance ranks among sites (=carrion patches; Fig. 6). Because our basic model (Eq. 13) estimates the degree of transitivity from the matrix of abundance ranks among sites, we expected to see a close link between both variables. Indeed, the average species abundances among all treatments increased with competitive strength (Fig. 5). However, the average coefficient of correlation in abundance ranks of the eight Diptera species among the 10 replicates per carrion weight class was only $\mathrm{r}_{\mathrm{S}}=0.30 \pm 0.13(\mathrm{p}<0.05)$, and average coefficient of correlation in abundance ranks of the five hymenopteran parasitoids was even weaker $\left(r_{S}=0.17 \pm 0.09, p>0.05\right)$. Therefore, we conclude that competitive transitivity is not a necessary prerequisite for stable abundance distributions among sites. In turn, it follows that our method is able to detect intransitive loops in competitive chains even when the resulting abundances distributions among sites are similar.

Predicted competitive hierarchies in Diptera and Hymenoptera were not stable among experimental treatments
(Table 1), or across generations of Hymenoptera (Table 3). However, Ulrich (1999) did not observe an increased number of parasitoid species with increasing host density. Therefore, biases introduced by possible effects of matrix sizes (more species) cannot explain the observed reordering of the competitive chains among slug weight classes. A possible trigger might have been the number of dipteran hosts available, which increased linearly with carrion weight (Ulrich 1999). At low host density, host availability is a limiting factor and priority effects - but not the survival of host larvae - should be important determinants of the competitive hierarchy. At high host density, priority effects might be less important, and we discovered a tendency towards more species spatial segregation at higher slug weight classes. Spatial segregation may diminish the impact of interspecific competition among coexisting species (Silvertown et al. 1999, Chesson 2000), which is consistent with our finding that competitive hierarchies were relaxed in cases of stronger species spatial segregation (Table 3).

Assemblages of necrophagous insects are not usually in equilibrium, and community patterns are often determined by food inputs (Beaver 1977, Hanski 1987). Priority effects at a suitable carcass largely determine the survival of larvae and the abundance of patches (Ulrich 1999), and therefore control the competitive hierarchy in a carrion patch. As a consequence, hierarchies vary among patches, which may enhance the regional coexistence of species (Allen et al. 1993). Thus, the detection of fully transitive relationships in combination with varying competitive rank order might be an indicator of both non-equilibrium conditions and priority effects. This interpretation is corroborated by the fact that intransitive hierarchies appeared only in the case of Hymenoptera colonizing the largest carrion weight classes. These large resource patches contain sufficient numbers of host larvae to reduce priority effects in favor of other mechanisms, including differential larval mortality and predation (Peschke et al. 1987).

\section{Summary}

To the best of our knowledge, our tests represent the first tools available for estimating the degree of intransitivity in competitive networks from replicated samples of species abundance, a common form of community data. Benchmark tests with artificial matrices revealed that these metrics could successfully detect intransitive competition networks, even in the absence of direct measures of pairwise competitive strength. These methods can be applied to replicated temporal and spatial data sampled in homogeneous environments or across environmental gradients, and can be applied to experimental measurements of pairwise interactions when they are available.

Acknowledgements - WU was in part supported by grants from the Polish Science Ministry (KBN, 3 P04F 034 22, KBN 2 P04F 039 29). SS and FTM were supported by the European Research Council under the European Community's Seventh Framework Programme (FP7/2007-2013)/ERC Grant agreement no. 242658 (BIOCOM). NJG was supported by the US National Science Foundation (NSF DEB-0541936) and the US Department of Energy (022821). 


\section{References}

Allen, J. C. et al. 1993. Chaos reduces species extinction by amplifying local population noise. - Nature 364: 229-232.

Allesina, S. and Levine, J. M. 2011. A competitive network theory of species diversity. - Proc. Natl Acad. Sci. USA 108: 5638-5642.

Baltzer, H. et al. 1998. Cellular automata models for vegetation dynamics. - Ecol. Modell. 107: 113-125.

Beaver, R. A. 1977. Non-equilibrium 'island' communities: Diptera breeding in dead slugs. - J. Anim. Ecol. 46: 783-798.

Bowker, M. A. and Maestre, F. T. 2012. Inferring local competition intensity from patch size distributions: a test using biological soil crusts. - Oikos 121: 1914-1922.

Bowker, M. A. et al. 2010. Competition increases with abiotic stress and regulates the diversity of biological soil crusts. - J. Ecol. 98: 551-560.

Callaway, R. M. 2007. Positive interactions and interdependence in plant communities. - Springer.

Chesson, P. 2000. Mechanisms of maintenance of species diversity. - Annu. Rev. Ecol. Syst. 31: 343-366.

Connell, J. H. and Slatyer, R. O. 1977. Mechanisms of succession in natural communities and their role in community stability and organization. - Am. Nat. 111: 1119-1144.

Diamond, J. M. 1975. Assembly of species communities. - In: Cody, M. L. and Diamond, J. M. (eds), Ecology and evolution of communities. Harvard Univ. Press, pp. 342-444.

Engel, E. C. and Wetzin, J. F. 2008. Can community composition be predicted from pairwise species interactions? - Plant Ecol. 195: 77-85.

Fox, J. W. 2013. The intermediate disturbance hypothesis should be abandoned. - Trends Ecol. Evol. 28: 86-92.

Fugère, V.et al. 2012. Testing the stress-gradient hypothesis with aquatic detritivorous invertebrates: insights for biodiversity-ecosystem functioning research. - J. Anim. Ecol. 81: 1259-1267.

Gause, G. F. 1934. The struggle for existence. - Williams and Wilkins, Baltimore.

Gilpin, M. E. 1975. Limit cycles in competition communities. - Am. Nat. 109: 51-60.

Gotelli, N. J. 2008. A primer of ecology, 4th edn. - Sinauer.

Gotelli, N. J. and Graves, G. R. 1996. Null models in ecology. - Smithsonian Inst. Press.

Gotelli, N. J. and Ulrich, W. 2012. Statistical challenges in null model analysis. - Oikos 121: 171-180.

Grace, J. B. et al. 1993. The examination of a competition matrix for transitivity and intransitive loops. - Oikos 68: 91-98.

Hanski, I. 1987. Carrion fly community dynamics: patchiness, seasonality and coexistence. - Ecol. Entomol. 12: 257-266.

Horn, H. S. 1975. Markovian properties of forest succession. - In: Cody, M. L. and Diamond J. M. (eds), Ecology and evolution of communities. Harvard Univ. Press, pp. 196-211.

Hubbell, S. P. 2001. The unified neutral theory of biogeography and biodiversity. - Princeton Univ. Press.

Huisman, J. et al. 2001. Towards a solution of the plankton paradox: the importance of physiology and life history. - Ecol. Lett. 4: 408-411.

Kawai, T. and Tokeshi, M. 2007. Testing the facilitation competition paradigm under the stress-gradient hypothesis: decoupling multiple stress factors. - Proc. R. Soc. B 274: 2503-2508.

Keddy, P. A. and Shipley, B. 1989. Competitive hierarchies in herbaceous plant communities. - Oikos 54: 234-241.

Kerr, B. et al. 2002. Local dispersal promotes biodiversity in a real-life game of rock-paper-scissors. - Nature 418: 171-174.

Laird, R. A. and Schamp, B. S. 2006. Competitive intransitivity promotes species co-existence. - Am. Nat. 168: 182-193.
Laird, R. A. and Schamp, B. S. 2008. Does local competition increase the coexistence of species in intransitive networks? - Ecology 89: 237-247.

Laird, R. A. and Schamp, B. S. 2009. Species coexistence, intransitivity, and topological variation in competitive tournaments. - J. Theor. Biol. 256: 90-95.

Levine, J. M. 1999. Indirect facilitation: evidence and predictions from a riparian community. - Ecology 80: 1762-1769.

Levine, J. M. and Rees, M. 2002. Coexistence and relative abundance in annual plant assemblages: the roles of competition and colonization. - Am. Nat. 160: 452-467.

Lortie, C. J. et al. 2004. Rethinking plant community theory. - Oikos 107: 433-438.

May, R. M. and Leonard, W. J. 1975. Nonlinear aspects of competition between three species. - SIAM J. Appl. Math. 29: 243-253.

McAuliffe, J. R. 1988. Markovian dynamics of simple and complex desert plant communities. - Am. Nat. 131: 459-490.

Meserve, P. L. et al. 1996. Role of biotic interactions in a small mammal assemblage in semiarid Chile. - Ecology 77: $133-148$

Miller, T. E. and Werner, P. A. 1987. Competitive effects and responses between plant species in a first-year old field community. - Ecology 68: 1201-1210.

Ovaskainen, O. et al. 2010. Modeling species co-occurrence by multivariate logistic regression generates new hypotheses on fungal interactions. - Ecology 91: 2514-2521.

Paine, R. T. 1984. Ecological determinism in the competition for space. - Ecology 65: 1339-1348.

Peschke, K. et al. 1987. Ecological separation, functional relationships, and limiting resources in a carrion insect community. - Zool. Jahrb. Syst. 114: 241-265.

Petraitis, P. S. 1979. Competitive networks and measures of intransitivity. - Am. Nat. 114: 921-925.

Pritchard, J. K. et al. 2000. Inference of population structure using multilocus genotype data. - Genetics 155: 945-959.

Reichenbach, T. et al. 2007 Mobility promotes and jeopardizes biodiversity in rock-paper-scissors games. - Nature 448: 1046-1049.

Rojas-Echenique, J. R. and Allesina, S. 2011. Interaction rules affect species coexistence in intransitive networks. - Ecology 92: $1174-1180$.

Shipley, B. 1993. A null model for competitive hierarchies in competition matrices. - Ecology 74: 1693-1699.

Silvertown, J. and Dale, P. 1991. Competitive hierarchies and the structure of herbaceous plant communities. - Oikos 61: 441-444.

Silvertown, J. et al. 1999. Hydrologically-defined niches reveal a basis for species-richness in plant communities. - Nature 400: 61-63.

Soliveres, S. et al. 2011. Microhabitat amelioration and reduced competition among understorey plants as drivers of facilitation across environmental gradients: towards a unifying framework. - Persp. Plant Ecol. Evol. Syst. 13: 247-258.

Staniczenko, P. P. A. et al. 2013. The ghost of nestedness in ecological networks. - Nat. Commun. 4: 1391.

Stone, L. and Roberts, A. 1990. The checkerboard score and species distributions. - Oecologia 85: 74-79.

Tilman, D. 1988. Plant strategies and the dynamics and structure of plant communities. - Monogr. Popul. Biol. 26. Princeton Univ. Press.

Tilman, D. 1994. Competition and biodiversity in spatiallystructured habitats. - Ecology 75: 2-16.

Ulrich, W. 1999. Species composition, coexistence and mortality factors in a carrion-exploiting community composed of necrophagous Diptera and their parasitoids (Hymenoptera). - Pol. J. Ecol. 47: 49-72. 
Ulrich, W. 2001. Hymenopteren in einem Kalkbuchenwald: Eine Modellgruppe zur Untersuchung von Tiergemeinschaften und ökologischen Raum-Zeit-Mustern. - Schriftenreihe des Forschzentrums Waldökosysteme A 171. Göttingen.

Ulrich, W. 2011. Turnover - a Fortran program for the analysis of species associations. $-<$ www.keib.umk.pl>.

Ulrich, W. 2013. Transitivity - a Fortran program for the analysis of bivariate competitive interactions. - <www.keib.umk.pl $>$.

Ulrich, W. and Gotelli, N. J. 2010. Null model analysis of species associations using abundance data. - Ecology 91: 3384-3397.

Ulrich, W. and Gotelli, N. J. 2013. Pattern detection in null model analysis. - Oikos 122: 2-18.

Supplementary material (available as Appendix oik-01217 at $<$ www.oikosjournal.org/readres/appendix >). Supplement A: Source code of the Fortran software application Transitivity (Ulrich 2013; <www.keib.umk.pl/transitivity/>) used to calculate the test matrices and transitivity scores. Supplement B: Supplementary raw data of the case study. Supplement C: Worked example of the benchmark testing procedure.
Ulrich, W. et al. 2012. Null model tests for niche conservatism, phylogenetic assortment and habitat filtering. - Meth. Ecol. Evol. 3: 930-939.

Webb, C. O. et al. 2002. Phylogenies and community ecology. - Annu. Rev. Ecol. Syst. 33: 475-505.

Worm, B. and Karez, R. 2002. Competition, coexistence and diversity in rocky shores. - In: Sommer, U. and Worm, B. (eds), Competition and coexistence. Springer, pp. 133-163.

Zaplata, M. K. et al. 2013. Species-driven phases and increasing structure in early-successional plant communities. - Am. Nat. 181: E17-E27. 